\title{
From Isovists via Mental Representations to Behaviour: First Steps Toward Closing the Causal Chain
}

\begin{abstract}
This study addresses the interrelations between human wayfinding performance, the mental representation of routes, and the geometrical layout of path intersections. The virtual reality based empirical experiment consisted of a route learning and reproduction task and two choice reaction tasks measuring the acquired knowledge of route decision points. In order to relate the recorded behavioural data to the geometry of the environment, a specific adaptation of isovist-based spatial analysis was developed that accounts for directional bias in human spatial perception and representation. Taken together, the applied analyses provided conclusive evidence for correspondences between geometrical properties of environments as captured by isovists and their mental representation.
\end{abstract}

Keywords: Isovist - geometry - wayfinding - spatial cognition - landmark knowledge - route knowledge - virtual reality

\section{Introduction}

Although original space syntax measures mainly addressed relations between spatial structures and society, recently several researchers have applied these descriptions to quantify relations between environmental structures and individual behavioural responses [e.g., 14, 5, 35]. While the obvious success of these studies has backed this extension of the original scope of the analyses, conclusive explanations or insights into the mechanisms underlying these statistically observable patterns have not yet been provided. Unlike mindless agents, human beings normally do not solely respond to a given spatial stimulus, their navigation behaviour rather results from mental planning processes and the monitoring of goals, processes which are continuously updated according to the current perceptual context. Therefore, in order to proceed from the mere description of correlations between environmental structures and averaged spatial behaviour to qualified predictions and explanatory models, it seems necessary to determine the perceptual and mental processes underlying these behavioural patterns. As an initial step toward this long-term goal, this paper addresses the relevance of the geometrical information captured by isovists for mental representations.

In the following subsections, relevant literature regarding isovists and mental representations related to wayfinding is reviewed. In Section 2, we describe the experiment where participants learned and retraced two routes through a photorealistic virtual environment. Here also the methodology regarding isovists and mental representations is introduced. In Section 3 the results are presented. We 
discuss them in Section 4 with respect to literature both from the domain of spatial analysis and from the area of spatial cognition.

\subsection{Space Syntax, Isovists, and Visibility Graphs}

Space syntax is a set of technologies for the analysis of spatial configurations using simple graphs solely consisting of paths and nodes $[16,17,18]$. The techniques were developed in the late 1970 in order to analyze interrelations between spatial and social structures. This analytical reduction of space to mere topological mathematical information facilitates the calculation of characteristic values and the quantitative comparison of environments. Originally, space syntax was developed to analyze topological properties of large-scale spatial configurations from the room layout of building complexes to whole cities. Hence, these techniques deliberately abstracted from geometrical detail.

For analyzing geometry-related spatial characteristics of environments, Benedikt [2] proposed isovists as objectively determinable basic elements. Isovists capture local spatial properties by collapsing the space visible from a single observation point to its two-dimensional abstraction. From these viewshed polygons, several quantitative geometrical descriptors can be derived such as area, perimeter length, or number of vertices. In a second step, these values can be mathematically combined to get further characteristic values. In order to better describe the geometry and also configurational characteristics of an environment as a whole, Turner, Doxa, O'Sullivan, \& Penn [33] have developed the technique of visibility graph analysis that combines aspects of global space syntax graphs with local intervisibility information as captured by isovists. Furthermore, this technique lends itself well for computer implementations. Although isovists are an abstract geometrical abstraction, recent research has shown that isovists are correlated with spatial behaviour and affective responses to indoor spaces [e.g., 10, 32, 35].

Isovists basically describe local geometrical properties of spaces with respect to individual observation points and weight all possible view directions equally. Especially for the analysis of individual motion trajectories, sometimes also viewspecific partial isovists have been applied [e.g., 4]. Partial isovists consider only a restricted part of the theoretically available visual field (e.g., $90^{\circ}$ instead of $360^{\circ}$ ). They correspond better to the restrictions of the human visual apparatus. Analogously, several studies have shown that humans encode spatial information from the point of view they encounter it [e.g., 3, 6, 11, 23].

Isovists are means to describe aspects of the outside world. As our goal is to reveal a connection between the geometric properties of the outside world and the inside world, we will now look what we store in our heads when walking around.

\subsection{Knowledge in Wayfinding}

In the wayfinding literature the distinction between landmark, route, and survey knowledge has received a lot of attention [e.g., 12, 15, 22, 27, 30, 31]. Landmarks are salient locations in the human environment such as a church or a square. Landmark 
knowledge refers to the recognition of these locations, e.g., "I know this esplanade, so I've been here before". Landmark knowledge alone is not sufficient to reach a goal. By recognizing a landmark, we know that we are on the right track, this however does not tell us, where to go next. The correct movement decision at an identified location requires route knowledge. Route knowledge describes the path that one must walk to reach the goal by telling the individual what to do at the decision points on the route, e.g., turn right at the church, then the second street to the left. It is one-dimensional or "string-like" and does not necessarily involve the knowledge of the exact location of the goal. Survey knowledge, in contrast, provides the direction and distance a location is to be found independent from knowing a path which leads there, e.g., the train station is about 300 Meters east from here. It is two-dimensional or "map-like". As survey knowledge is not route specific it will not be regarded further in this paper.

\subsection{Predictions}

Landmark and route knowledge together with wayfinding performance will be the dependant measures of our study. The different geometries of intersections expressed by isovist measures will be the independent measures of our study. Our prediction is that there is a connection not only between the geometry of intersections and wayfinding performance, but also between the geometry and mental representations, namely landmark and route knowledge.

\section{Methods}

For the experiment we used a virtual environment displayed on a $220^{\circ}$ semicylindrical screen. The participants learned two different routes through "Virtual Tübingen" a photorealistic model of the medieval city centre of Tübingen (see Fig. 1, [34]). Directly after learning a route, participants had to find and to "virtually walk" this route with a joystick. After that we measured the acquired landmark and route knowledge with two choice reaction tasks. In order to represent expected directional biases, the isovist analysis made use of partial isovists capturing the perspectives seen when approaching the intersections. We validated this approach in the landmark knowledge task by comparing different perspectives on the intersections. Eleven isovist statistics were used to classify the intersections in two geometrically dissimilar groups. Then we compared the wayfinding performance and knowledge in these two groups of intersections. 


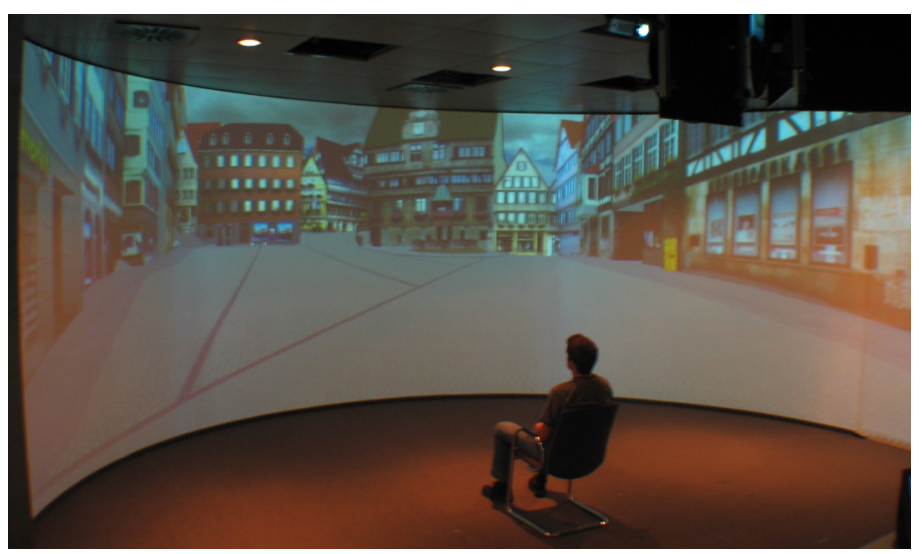

Fig. 1. The setup for learning and navigating the routes in Virtual Tübingen.

\subsection{Knowledge and wayfinding performance}

Participants. Twelve female and twelve male participants, mainly students between 19 and $32(M=24 ; S D=4)$, participated in the experiment. None of them had visited Tübingen before. All selected participants were German native speakers and were paid for their participation. Two of original 26 participants did not complete the experiment due to simulator sickness and were therefore excluded from all subsequent analysis.

Learning the Routes and Wayfinding performance. The participants sat on a chair positioned at the focal point 3.5 meters away from a circular $220^{\circ}$ screen (width: $13 \mathrm{~m}$, height: $3 \mathrm{~m}$ ), which covered the whole horizontal visual field (see Fig. 1). A pc-cluster rendered the projection for an eye position 1.20 meter above the ground referring to an average eye-height in a seated position. The scene was rendered at a frame rate of $60 \mathrm{~Hz}$ using $2 \mathrm{x}$ hardware anti-aliasing and hardware correction to display the images geometrically correct on the curved screen. Three projectors with a resolution of 1024 $\mathrm{x} 768$ each projected the pictures.

For learning the routes the participants were passively carried through the environment. The transportation speed was two meters per second corresponding to a fast walking speed. The long route spanned 480 meters and consisted of ten mainly oblique intersections with 23 possible choices (see Fig. 2). Having a length of 320 meters, the short route contained nine mainly orthogonal intersections offering altogether 21 possible direction choices (for a further description of these routes see [24]). The order of presentation of the routes was controlled. During route learning, participants were confronted with either a verbal, a visual, a spatial, or no secondary task. This aspect of the experiment is described in more detail in Meilinger, Knauff and Bülthoff $[25,26]$. 


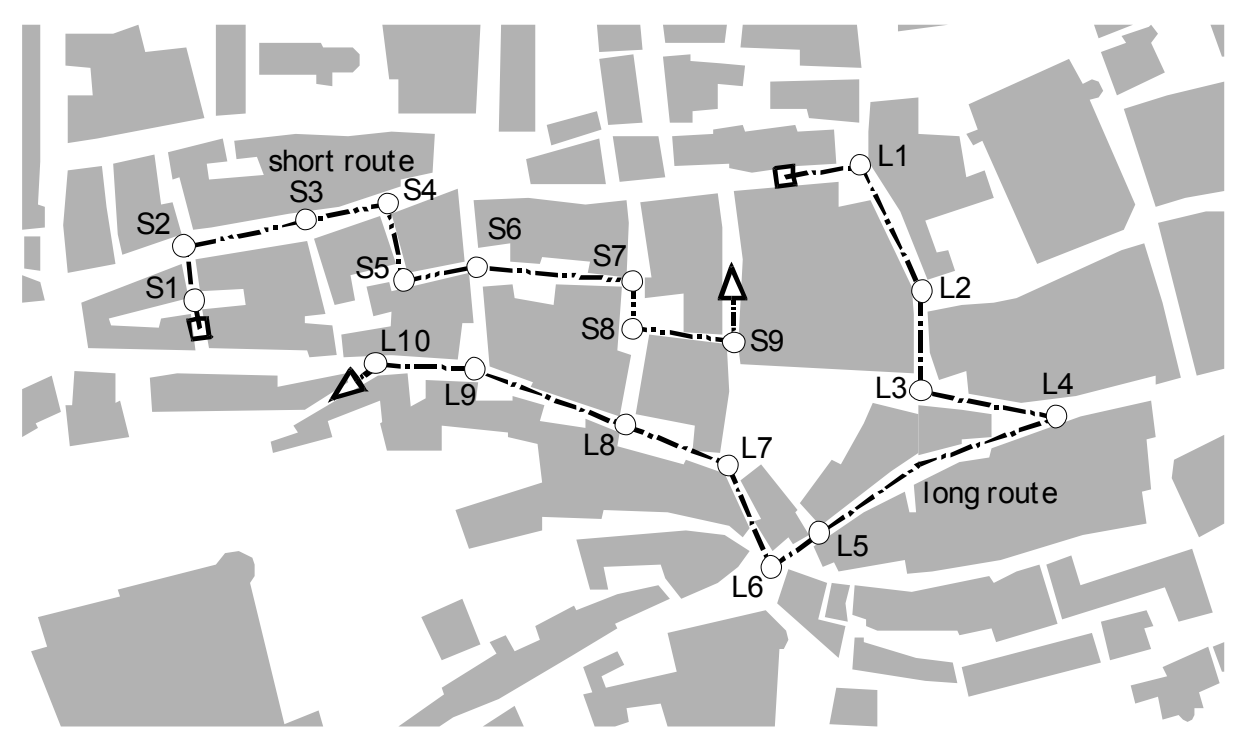

Fig. 2. The two routes through Virtual Tübingen used in the experiment.

No secondary task was applied when the participants actively navigated the routes immediately afterwards. Therefore, all participants had the chance to acquire knowledge without being distracted by a secondary task. During navigation, participants could control their heading and forward translation speed using a customary joystick device. The maximal translation speed was two meters per second. In order to reduce simulator sickness, rotation speed was restricted to $30^{\circ}$ per second. The dependent variable wayfinding performance was measured by the proportion of correct route choices at specific intersections. When participants chose an incorrect route continuation, they were stopped after about 5 meters by the simulation. In this case they had to turn around in order to continue their navigation.

Before the experiment, participants were familiarized with the virtual reality setup and the joystick-based interaction in an area of Virtual Tübingen not encountered during the rest of the experiment.

Test of landmark knowledge. We measured landmark knowledge for intersections in a choice reaction task. Pictures of all intersections sized 1024 x 786 pixel were presented on a screen. In the pictures, the facades of houses situated in front of the intersection were visible (see Fig. 3, left side). Participants had to press a button on a response box as fast as possible to indicate whether they had seen the intersection before. The same procedure was also used to test the perspective bias in recognizing intersections (see Section 2.2). The pictures presented were taken from every street approaching an intersection. So for a four arm intersection, four pictures had to be judged. 61 pictures of intersections and 8 distracters were presented this way. The distracters were pictures taken from intersections in virtual Tübingen not previously seen by the participants. All pictures were presented in random order. The positions of the hit and reject buttons on the response box were selected randomly for each participant. Accuracy and reaction times were recorded. Extreme values deviating 
more than three standard deviations from the mean were replaced by the most extreme value observed within three standard deviations.

Test of route knowledge. A choice reaction task was used to measure route knowledge. Pictures of intersections were presented, participants had to indicate the correct route continuation by deflecting a joystick in the correct direction as fast as possible (see Fig. 3, right side). In case they were not able to recognize the intersection, they were instructed to deflect the joystick in a backward direction. The pictures used in the route knowledge test phase were identical to the pictures in the landmark knowledge task, but exclusively perspectives along the direction of travel were used. 19 pictures of intersections and 4 distracters were presented this way. Other distracters than in the landmark knowledge task were used that were also pictures from intersections not previously seen by the participants. Pictures and distracters were presented in random order separated by routes. Each picture and distracter was presented twice. Accuracy and reaction times were recorded. The correction of extreme values was identical like in landmark knowledge.
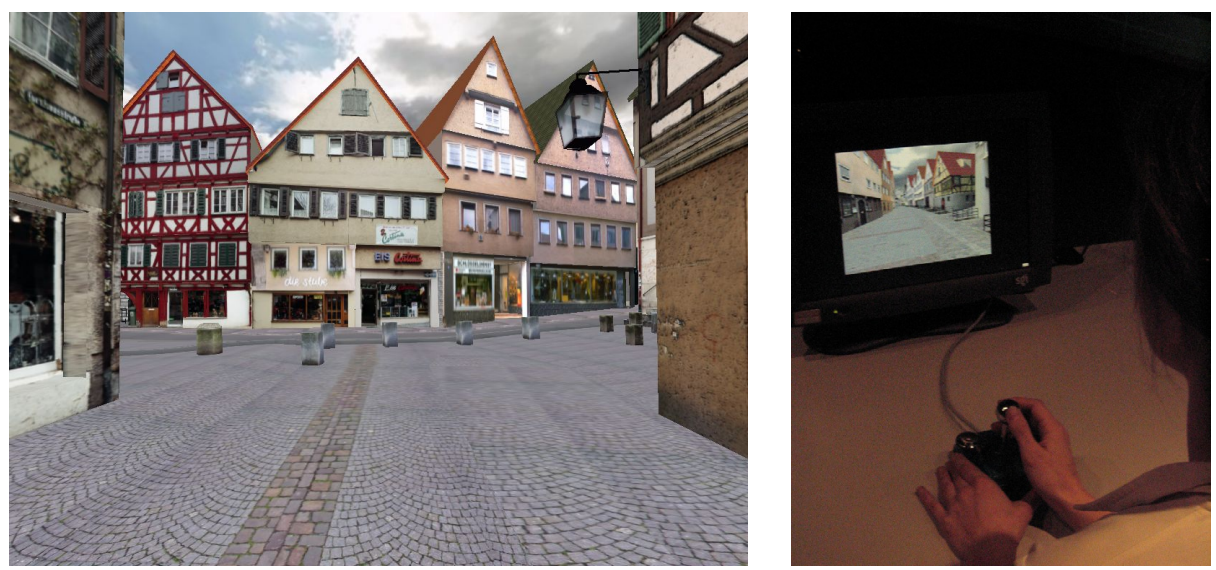

Fig. 3. To measure their landmark and route knowledge, participants saw pictures like the one on the left side. For route knowledge the participants indicated the further route with a joystick as seen on the right side.

\subsection{Test of Perspective-dependent and Geometry-dependent Recognition Biases}

We wanted to test whether the directed route presentation and exploration in the initial learning phase of the experiment led to a stronger memorization of this particular perspective. Therefore, we analyzed the data obtained from the landmark recognition task (see landmark knowledge) on direction-specific differences. For this purpose, the performance in discriminating a picture of an intersection from a distracter $d$ ' was computed for each perspective of an intersection [13]. The statistic $d$ ' expresses the difference between the normal distribution of stimuli and the normal distribution of distracters in standard deviations. A $d$ ' of 1.0 means that the two 
distributions are one standard deviation apart. If a participant recognized all distracters or targets, $d$ ' could not be computed. In this case a recognition rate of $100 \%$ was replaced by a $99 \%$ score. The perspective seen when approaching the intersection was expected to be recognized more easily compared to perspectives in a $90^{\circ}$ or $180^{\circ}$ angle to this perspective. Reaction times and $d^{\prime}$ in these groups of pictures were compared within-subject using an ANOVA with post-hoc t-tests.

\subsection{The Direction-Specific Isovist Analysis}

Isovists. The differential analysis between intersections described above required a quantitative description of the individual intersections. In order to test whether expected differences could be attributed to some visuo-spatial properties, a quantitative description of the intersections' geometrical layout and shape based on isovists was calculated. Isovists, as originally conceived by Benedikt [2], equally describe all possible view directions from a given single observation point, a perspective which is directly perceptible only in an unnatural bird's eye view of a spatial environment. In reality, however, observers experience the environment mainly from a directed inside perspective along their main line of travel, suggesting a different weighting of view directions depending on their relative angle to this main direction. In order to account for this in the isovist-based spatial analysis, two specific adaptations were introduced: First, instead of basing the analysis on ordinary $360^{\circ}$ isovists, directed partial isovists spanning a horizontal angle of $90^{\circ}$ were applied [cf. 4]. Second, in order to include also information on branchings beyond this restricted angle, the reference points of the isovists were shifted from the center of the road intersection in approach direction. Thus the isovists corresponded to the visual field as available immediately before entering the junctions. (cf. Fig. 3 left side).

The eleven isovist-based geometrical descriptors of the junctions were calculated using the free ajanachara tool [9] which offers both isovist and visibility graph-based statistics. The analysis was done at a spatial resolution of 1.5 meters. Table 1 gives a short overview of the individual variables which comprised typical local geometrical measures from the isovist literature. For more detailed information, please refer to Franz and Wiener [8].

Isovist-based Categorization of Intersection. Based on the eleven isovist statistics obtained by the analysis described in the previous section, a measure of geometrical similarity of the intersections was calculated. Since isovist statistics typically correlate highly with each other, first, a factor analysis was applied to identify independent dimensions underlying these parameters [e.g., 1, 21]. A principal component analysis extracted factors with an eigenvalue $>1.0$ out of the correlation matrix. In order to do so, the isovist statistics were correlated with each other over the intersections. A multiple linear regressing estimated the communalities. The resulting factor matrix was rotated using the VARIMAX method. Each intersection could be described now by their factor values on three independent factors. A hierarchical cluster analysis grouped the intersections on basis of these factor values using Euclidean distances 
and the Ward method to compute distances between groups of intersections [e.g., 1, 7]. The last two groups of intersections to be clustered together were taken as geometrically distinctive groups of intersections. To see if participants reacted differently to these geometric layouts, navigation performance, landmark knowledge, and route knowledge on these two groups of intersections were compared with each other in t-tests.

Table 1. Description of the eleven isovist statistics used in the analysis comparing the geometrical characteristics of the junctions.

\begin{tabular}{|c|c|}
\hline Isovist Statistic & Short Description \\
\hline Area & $\begin{array}{l}\text { Number of } 1.5 \mathrm{~m} \times 1.5 \mathrm{~m} \text { squares lying with at least } 50 \% \text { inside the } \\
\text { isovist }\end{array}$ \\
\hline Perimeter length & Overall length of the isovist boundary \\
\hline Vertices & Number of vertices of the isovist polygon \\
\hline $\begin{array}{l}\text { Vertices per } \\
\text { perimeter }\end{array}$ & Number of vertices divided by perimeter \\
\hline Vertices per area & Number of vertices divided by area \\
\hline Roundness & Isovist area divided by squared perimeter length \\
\hline Jaggedness & Squared Perimeter length divided by area \\
\hline $\begin{array}{l}\text { Bounding } \\
\text { proportion }\end{array}$ & $\begin{array}{l}\text { Length of the principal axis of a minimal bounding rectangle divided by } \\
\text { its secondary axis }\end{array}$ \\
\hline Convexity & $\begin{array}{l}\text { Roundness divided by bounding properties. A measure for the deviation } \\
\text { of the isovist from a rectangle }\end{array}$ \\
\hline Openness & $\begin{array}{l}\text { Length of open edges dived by length of closed edges. Closed edges are } \\
\text { visible walls, open edges result from occlusions }\end{array}$ \\
\hline Clustering & Percentage of pairs of squares in the isovist which can see each other \\
\hline
\end{tabular}

\section{Results}

\subsection{Perspective-bias in Recognition}

To tell whether the perspective seen when approaching an intersection was the most relevant, different perspectives of intersections were compared in the landmark knowledge task. We computed the performance in discriminating the different perspectives of intersections from the distracters. The performances differed due to the angle between the perspective the picture was taken and the direction of traveling (see Fig. 4; $d$ ': $F(2,46)=29.8, p<.001, \eta^{2}=.56$; reaction time: $F(2,46)=12.8, p<$ $\left..001, \eta^{2}=.36\right)$. Pictures taken along the direction of traveling $\left(0^{\circ}\right)$ were recognized better compared to pictures taken from $90^{\circ}$ to that $\left(d^{\prime}: t(23)=10.2, p<.001\right.$, effect size $=2.08$; reaction time: $t(23)=4.12, p<.001$, effect size $=0.84$ ) or taken from $180^{\circ}\left(d^{\prime}: t(23)=3.84, p<.001\right.$, effect size $=0.78$; reaction time: $t(23)=4.42, p<$ .001 , effect size $=0.90)$. Pictures taken from $90^{\circ}$ were recognized worse than pictures taken from $180^{\circ}\left(d^{\prime}: t(23)=3.05, p=.006\right.$, effect size $=0.62$; reaction time: $t(23)=$ $1.15, p=.262$, effect size $=0.23$ ). 

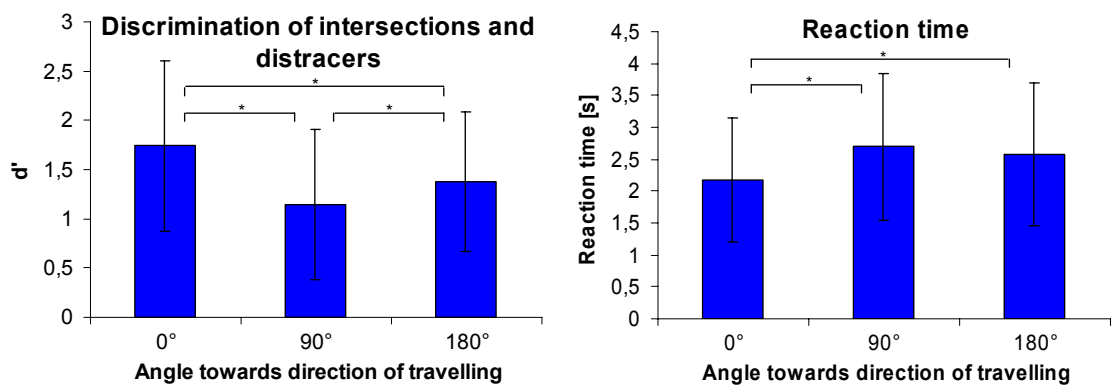

Fig. 4. d' values expressing the performance of differentiation between distracters and pictures of intersections (left) and reaction times (right). The pictures of the intersections were taken from the direction the intersections were approached originally $\left(0^{\circ}\right)$ or from an angle of $90^{\circ}$ or $180^{\circ}$ to that direction. Means and standard deviations are displayed. Asterisks mark significant differences at $p<.05$.

\subsection{Isovist Analysis}

We used an isovist analysis to identify two groups of geometrically different intersections and relate them to navigation performance and knowledge measures. The space visible when approaching an intersection was expressed in eleven isovist statistics. A principal component analysis identified three independent factors with an eigenvalue $>1$ underlying the eleven highly correlated isovist measures (see Table 2). Geometrically similar intersections show similar isovist statistics and therefore also similar values on the underlying factors.

Table 2. The rotated component matrix with the loadings of the isovist statistics on the three independent factors. Grey shading indicate higher loadings. This means that the factor expresses much of the variance of this isovist statistic.

\begin{tabular}{lccc}
\hline & Factor $\mathbf{1}$ & Factor 2 & Factor 3 \\
\hline Vertices & 0,89 & $-0,11$ & $-0,13$ \\
Bounding properties & 0,88 & $-0,24$ & $-0,03$ \\
Convexity & $-0,76$ & 0,35 & 0,46 \\
Area & 0,67 & $-0,57$ & 0,18 \\
Perimeter & 0,69 & $-0,62$ & $-0,21$ \\
Roundness & $-0,65$ & 0,54 & 0,50 \\
Vertices per perimeter & $-0,28$ & 0,92 & 0,16 \\
Vertices per area & $-0,26$ & 0,93 & 0,01 \\
Clustering & $-0,12$ & 0,37 & 0,84 \\
Openness & $-0,01$ & 0,33 & $-0,82$ \\
Jaggedness & 0,51 & $-0,45$ & $-0,64$ \\
\hline
\end{tabular}


A hierarchical cluster analysis grouped the intersections successively based on their geometrical similarity expressed in similar values in theses three independent factors. First, very similar single intersections were grouped together. Then, similar groups were merged together until in the end only two groups remained before being merged together (see Fig. 5). These last two groups consisted of T-intersections that are the intersections S5, S7, S9, L1, L3 and L4 in contrast to the non-T-intersections. The performance on theses two groups of geometrically different intersections was compared. At non-T-intersections the participants clearly performed better than at Tintersections (see Table 3). The participants recognized non-T-intersections faster than T-intersections $(t(22)=2.51, p=.020$; accuracy $t(23)=1.21, p=.238)$. At non$\mathrm{T}$-intersections the accuracy in indicating the further route was higher compared to $\mathrm{T}$ intersections $(t(23)=4.71, p<.001$; reaction time $t(22)=0.76, p=.457)$. At Tintersections the participants got lost more often than at non-T-intersections $(t(23)=$ $2.56, p=.017)$. The geometry of intersections was associated not only with different wayfinding performance but also with different landmark and route knowledge.

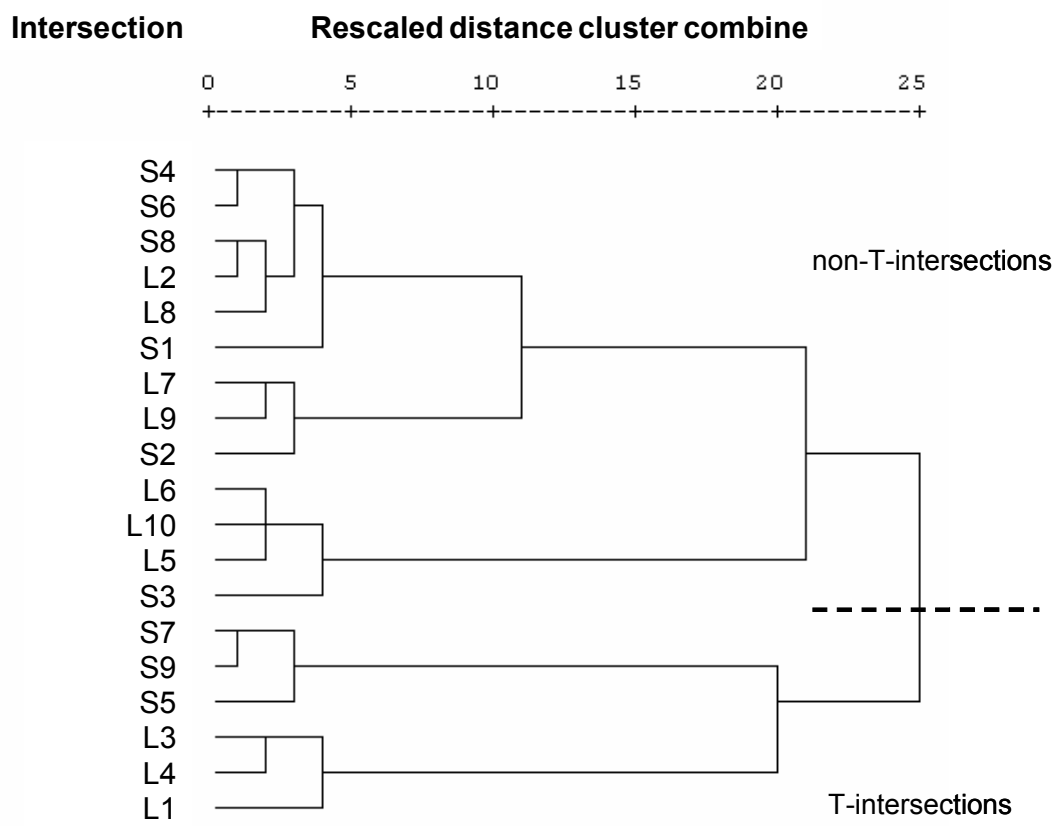

Fig. 5. Dentrogram of the hierarchical cluster analysis. Vertically all 19 intersections of the two routes are displayed. To the right is the Euclidian distance between intersections or groups of intersections in the three-dimensional space created by the three independent factors. Intersections or groups of intersections are grouped together at a certain Euclidian distance. Geometrically similar intersections are grouped at short distances, dissimilar ones at large distances. 
Table 3. Mean performance (with standard deviations) at $\mathrm{T}$ and non-T-intersections and effect sizes for the differences. Asterisks mark significant differences at $p<.05$.

\begin{tabular}{|c|c|c|c|}
\hline & T-intersections & $\begin{array}{c}\text { Non-T- } \\
\text { intersections }\end{array}$ & $\begin{array}{l}\text { Effect } \\
\text { size }\end{array}$ \\
\hline \multicolumn{4}{|l|}{ Landmark knowledge } \\
\hline Accuracy & $0.55(0.20)$ & $0.62(0.22)$ & 0.25 \\
\hline Reaction time* $[\mathrm{s}]$ & $2.62(1.43)$ & $2.22(1.03)$ & 0.52 \\
\hline \multicolumn{4}{|l|}{ Route knowledge } \\
\hline Accuracy* & $0.42(0.21)$ & $0.61(0.16)$ & 0.96 \\
\hline Reaction time $[\mathrm{s}]$ & $2.51(1.01)$ & $2.41(0.97)$ & 0.16 \\
\hline \multicolumn{4}{|c|}{ Wayfinding performance per intersection* } \\
\hline & $0.19(0.18)$ & $0.12(0.13)$ & 0.52 \\
\hline
\end{tabular}

\section{Discussion}

The present study examined the connection between geometrical properties of our environment and mental representations of this environment. The main finding is that geometrical properties are not only connected with directly observable wayfinding behaviour [e.g., 14, 5, 35], but that they are also connected with mental representations of this environment. T-intersections and non-T-intersections were the geometrically most dissimilar subgroups of intersections as revealed by isovist statistics. At T-intersections participants performed worse in the active navigation task as well as in the landmark and route knowledge tasks.

What could be reasons for this difference between $\mathrm{T}$ and non-T-intersections? Generally, T-intersections might be geometrically more similar with each other than non-T-intersections which could be branch-offs, cross-intersections or even more complex intersections. A higher similarity might lead to more confusions and therefore to a lower performance in wayfinding as well as landmark and route knowledge (cf. Fig. 6).

For both route knowledge and navigation performance, the observed better performance at non-T-intersections must be a very robust effect. In both measures, participants had to choose between alternatives. With more alternatives the task gets more difficult to solve by guessing [cf. 28, 29]. At non-T-intersections, the participants had to choose between 2.4 alternative routes in average whereas at $\mathrm{T}$ intersections the participants only had to choose between 2 alternatives. Despite this higher chance level at non-T-intersections, participants performed better, indicating a strong effect even overriding this bias. 

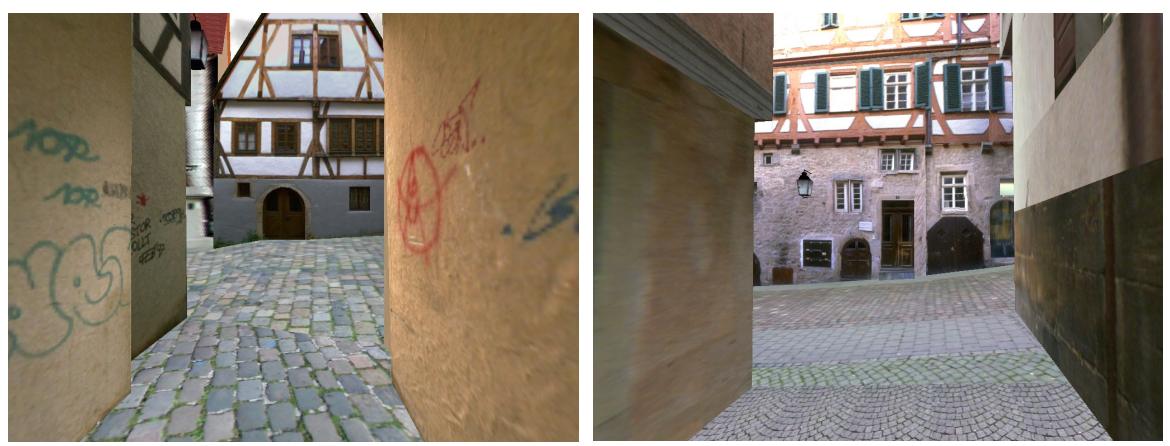

Fig. 6. Two T-intersections on the short route. At the left intersection (S7) a participant had to turn to the right or to indicate so in the route knowledge task. At the right intersection (S9) a participant had to turn to the left.

A second important point of this study is the inclusion of perspectivity in the isovist analysis. First, we did not apply isovist statistics with a $360^{\circ}$ field of view as is most commonly done, but limited the field of view by applying partial isovists [cf. 4]. Second, the isovists' reference points were shifted towards the approach direction. This approach is in accordance with anatomical constraints of the human visual apparatus and directly corresponds to the directional route presentation. It is in accordance with studies showing that humans encode spatial information from the point of view they encounter them, at least for environments not too familiar [e.g., 3, $6,11,23]$. In addition, we validated this approach by comparing the recognition performance of intersections. Analogous to the directional bias in the analysis, participants recognised intersections best when shown a picture taken along the direction of traveling. If perspectivity did not matter participants should have recognized the intersections equally well from all perspectives. Although the optimal angular size of partial isovists is object to future studies, one important conclusion can be drawn: As captured by the applied method, a T-intersection is psychologically different from a topologically equivalent branch off. This holds true also if the geometry of both intersections is identical.

In order to close the gap between isovist statistics and wayfinding behaviour by accounting for perception and mental representations, the correct consideration of perspectivity seems crucial. The acquisition of mental representations, however, is only one part of what happens in the brain during wayfinding. In order to make use of this information, the brain has to process these representations. Several strategies and heuristics how to process these representations have been proposed, e.g., the leastangle strategy [e.g., 19]. Other strategies like hierarchical fine-to-coarse planning [36] or sticking to well-known areas as much as possible have been proposed [20]. Based on the outcomes of this study, this multitude of strategies can be complemented by another heuristic which could be informally termed 'when-in-doubt-follow-yournose'. We compared the performance at intersections where participants had to walk straight on with those intersections which required a turning. Participants recalled these two groups of intersections equally well (see Table 4 landmark knowledge; accuracy: $t(23)=0.65, p=.520$; reaction time: $t(22)=1.10, p=.282)$. When asked to draw the routes including all intersections, they made less errors at drawing 
intersections which required a turn than at drawing intersections where the route went straight on $(t(23)=3.52, p=.002)$. Despite the equal to better memory for intersections requiring a turn, participants performed better at "straight-on" intersections when they had to decide for the further route. Participants correctly indicated to walk straight on more often than they indicated a correct turn (see Table 4 route knowledge; accuracy: $t(23)=3.44, p=.002$; reaction time: $t(23)=1.51, p=$ $.145)$. They also got lost less often at intersections where no turn was required $(t(23)=$ $3.58, p=.002$ ). We think that participants decided to walk straight on when they did not remember the further route. This 'when-in-doubt-follow-your-nose' strategy can reduce memory demands. Thus, participants only had to store and recall changes in the direction of travel. It was not necessary to recall where to go at straight-on intersections, because here the default strategy of walking straight on applies. In principle, one alternative explanation would be that participants had to walk straight on most of the times and that these results are therefore specific for these routes. This explanation could not hold true as participants had to walk straight on less often (7 times) than they were required to turn (12 times).

We described the 'when-in-doubt-follow-your-nose' strategy for retracing a route and for the memory of a route. The tendency of walking straight on has already been described for exploring an unknown virtual environment [4]. Here participants rather walk straight on than turn at an intersection.

Table 4. Mean performance (with standard deviations) at intersections where to walk straight on or with a turn required. Asterisks mark significant differences at $p<.05$.

\begin{tabular}{lccc}
\hline & $\begin{array}{c}\text { Route goes } \\
\text { straight on }\end{array}$ & Turn required & $\begin{array}{c}\text { Effect } \\
\text { size }\end{array}$ \\
\hline Errors at drawing intersections & & & \\
& $4.4(2.0)$ & $2.5(2.4)$ & 0.72 \\
Landmark knowledge & & & \\
$\quad$ Accuracy & $0.58(0.26)$ & $0.61(0.17)$ & 0.13 \\
$\quad \begin{array}{l}\text { Reaction time [s] } \\
\text { Route knowledge }\end{array}$ & $2.22(1.11)$ & $2.39(1.17)$ & 0.23 \\
$\quad$ Accuracy* & $0.65(0.18)$ & $0.49(0.18)$ & 0.70 \\
$\quad$ Reaction time [s] & $2.29(1.06)$ & $2.52(0.95)$ & 0.31 \\
Wayfinding performance per intersection* & & \\
\multicolumn{2}{l}{$0.06(0.09)$} & $0.19(0.18)$ & 0.73 \\
\hline
\end{tabular}

We presented various results in this paper. When interpreting and generalizing these results, one has to take especially two aspects into account. First, the results may not be interpreted causally. Not only geometry, but also any other environmental property correlated with geometry could be a relevant cause for the observed differences. Second, the experiment took place in a typical European city centre with lots of different intersections. The results might be limited to such geometrically rich environments. In a typical American rectangular grid like city layout with geometrical very similar intersections, geometry might play a less important role for wayfinding. 


\section{Conclusions}

Confirming the outcomes of many other studies, this paper has shown that isovist analysis is a powerful tool for quantitatively capturing behaviourally relevant geometric properties of environments. Beyond this, the presented study demonstrated for the first time correspondences between mental representations and geometric properties captured by isovists. Furthermore, this paper pointed towards the importance of perspectivity when predicting human behaviour. Although a street branching-off and a T-intersection might be identical in their abstract geometric and topological layout, they are different psychologically. Considering perspectivity, as in the conducted analysis, is one important point when closing the gap between an isovist analysis on one hand and predicted behaviour on the other hand. We are convinced that this gap can only be closed when taking mental representations and processes into account. The authors hope that this approach is a step not only towards closing the gap between space syntax analysis and behaviour but also towards narrowing the gap between architecture and spatial cognition.

Acknowledgments. The research was supported by grants from the DFG (German National Research Foundation) in the Transregional Collaborative Research Center, SFB/TR 8 project and by the EU grant "Wayfinding" ( $6^{\text {th }}$ FP - NEST). The authors thank Markus Knauff, Anna Widiger, Michael Weyel, Hans-Günther Nusseck, Harald Teufel, Benjamin Turski for their support.

\section{References}

1. Backhaus, K., Erichson, B., Plinke, W. \& Weiber, R. (1990). Multivariate Analysemethoden: Eine anwendungsorientierte Einführung. Berlin: Springer.

2. Benedikt, M. L. (1979). To Take Hold of Space: Isovists and Isovists Fields. Environment and Planning $B, 6,47-65$.

3. Christou, C. \& H.H. Bülthoff (1999). View dependence in scene recognition after active learning. Memory \& Cognition ,27, 996-1007.

4. Conroy, R. (2001). Spatial navigation in immersive virtual environments. Unpublished doctoral dissertation, University of London.

5. Conroy-Dalton, R. (2003). The secret is to follow your nose: Route path selection an angularity. Environment and Behavior, 35(1), 107-131.

6. Diwadkar, V. A., \& McNamara, T. P. (1997). Viewpoint dependence in scene recognition. Psychological Science, 8, 302-307.

7. Everitt, B.S. (1993). Cluster analysis. London: Edward Arnold.

8. Franz, G. \& Wiener, J.M. (2005). Exploring isovist-based correlates of spatial behavior and experience. Proceedings of the 5th Space Syntax Symposium, 503-517.

9. Franz, G. (2003). Ajanachara - a tool for visibility graph analysis. Retrieved January, 17, 2005, from Max-Planck-Instirute for Biological Cybernetics Web site: http://www.kyb.mpg.de/ gf/anavis

10. Franz, G., von der Heyde, M. \& Bülthoff, H.H. (2005). Predicting experiential qualities of architecture by its spatial properties. In B. Martens, B. \& A.G. Keul (Eds.), Designing 
Social Innovation: Planning, Building, Evaluating (pp. 157-166). Cambridge, MA: Hogrefe and Huber.

11. Garsoffky, B., Schwan, S. \& Hesse, F. W. (2002). Viewpoint dependency in the recognition of dynamic scenes. Journal of Experimental Psychology: Learning, Memory, and Cognition, 28, 1035-1050.

12. Golledge, R.G. (Ed.) (1999). Wayfinding behavior: Cognitive mapping and other spatial processes. Baltimore: The Johns Hopkins University Press.

13. Green, D.M. \& Swets, J.A. (1966). Signal Detection and Psychophysics. New York: Wiley.

14. Haq, S., \& Zimring, C. (2003). Just down the road a piece: The development of topological knowledge of building layouts. Environment and Behavior, 35(1), 132-160.

15. Herrmann, T., Schweizer, K., Janzen, G. \& Katz, S. (1998). Routen- und Überblickswissen - konzeptionelle Überlegungen. Kognitionswissenschaft, 7, 145-159.

16. Hillier, B. (1996). Space is the Machine. Cambridge, MA: Cambridge University Press.

17. Hillier, B. (1998). The common language of space: a way of looking at the social, economic and environmental functioning of cities on a common basis. Retrieved July, 14, 2006. University College London Web site: http://www.spacesyntax.org/publications/commonlang.html

18. Hillier, B. \& Hanson, J. (1984). The Social Logic of Space. Cambridge, UK: Cambridge University Press.

19. Hochmair, H., \& Frank, A.U. (2002). Influence of estimation errors on wayfinding decisions in unknown street networks - analyzing the least-angle strategy. Spatial Cognition and Computation, 2(4), 283-313.

20. Hölscher, C., Meilinger, T., Vrachliotis, G., Brösamle, M. \& Knauff, M. (2005). Finding the Way Inside: Linking Architectural Design Analysis and Cognitive Processes. In: C. Freksa, M. Knauff, B. Krieg Brückner, B. Nebel and T. Barkowsky (Eds.), Spatial Cognition IV - Reasoning, Action, Interaction (pp.1-23). Berlin: Springer.

21. Kim, J.O. \& Mueller, C.W. (1978). Factor analysis: Statistical methods and practical issues. London: Sage Publications.

22. Kitchin, R.M. \& Freundschuh, S. (Eds.) (2000). Cognitive Mapping: Past, Present and Future. London: Routledge.

23. Mallot, H.A. \& Gillner, S. (2000). Route navigation without place recognition: What is recognized in recognition-triggered responses? Perception, 29, 43-55.

24. Meilinger, T. \& Knauff, M. (submitted). Ask for your way or use a map: A field experiment on spatial orientation and wayfinding in an urban environment.

25. Meilinger, T., Knauff, M. \& Bülthoff, H.H. (2006). Working memory in wayfinding - a dual task experiment in a virtual city. Proceedings of the 28th Annual Conference of the Cognitive Science Society.

26. Meilinger, T., Knauff, M. \& Bülthoff, H.H. (submitted). Working memory in wayfinding a dual task experiment in a virtual city.

27. Montello, D. R., Waller, D., Hegarty, M., \& Richardson, A. E. (2004). Spatial memory of real environments, virtual environments, and maps. In G. L. Allen (Ed.), Human spatial memory: Remembering where (pp. 251-285). Mahwah, NJ: Lawrence Erlbaum Associates.

28. O'Neill, M. J. (1991a). Effects of signage and floorplan configuration on wayfinding accuracy. Environment and Behavior, 23, 553-574.

29. O'Neill, M. J. (1991b). Evaluation of a conceptual model of architectural legibility. Environment and Behavior, 23, 259-284.

30. Piaget, J. \& Inhelder, B. (1967). The child's conception of space. New York: Norton.

31. Siegel, A.W. \& White, S.H. (1975). The development of spatial representations of largescale environments. In H.W. Reese (Ed.), Advances in child development and behaviour (Vol. 10). New York: Academic Press.

32. Turner, A. \& Penn, A. (1999). Making isovists syntactic: isovist integration analysis. Paper presented at the $2^{\text {nd }}$ International Symposium on Space Syntax, Brasilia. 
33. Turner, A., Doxa, M., O'Sullivan, D., \& Penn, A. (2001). From isovists to visibility graphs: a methodology for the analysis of architectural space. Environment and Planning B, 28(1), 103-121.

34. van Veen, H.A.H.C., Distler, H.K., Braun, S.J. \& Bülthoff, H.H. (1998). Navigation through a Virtual City: Using Virtual Reality Technology to Study Human Action and Perception. Future Generation Computer Systems, 14, 231-242.

35. Wiener, J. M. \& Franz, G. (2005). Isovists as a means to predict spatial experience and behavior. In C. Freksa, M. Knauff, B. Krieg-Brückner, B. Nebel, \& T. Barkowsky (Eds.), Spatial Cognition IV - Reasoning, Action, Interaction. International Conference Spatial Cognition 2004 (pp. 42-57). Berlin: Springer.

36. Wiener, J. M., Schnee, A., \& Mallot, H. A. (2004). Use and interaction of navigation strategies in regionalized environments. Journal of Environmental Psychology, 24, 475493. 